\title{
Publisher's Note: Bimetric Theory of Fractional Quantum Hall States [Phys. Rev. X 7, 041032 (2017)]
}

\author{
Andrey Gromov and Dam Thanh Son
}

(Q) (Received 6 February 2018; published 14 February 2018)

DOI: 10.1103/PhysRevX.8.019901

This paper was published online on 10 November 2017 with an error in the Acknowledgments on page 12. On page 12, the last sentence of the Acknowledgments should read as "A. G. was supported by NSF Grant No. DMR-1206648." The Acknowledgments have been corrected as of 8 February 2018. 